\title{
Si-Jun-Zi Decoction Treatment Promotes the Restoration of Intestinal Function after Obstruction by Regulating Intestinal Homeostasis
}

\author{
Xiangyang Yu, ${ }^{1}$ Zhigang Cui, ${ }^{2}$ Zhenli Zhou, ${ }^{1}$ Tao Shan, $^{1}$ Donghua Li, ${ }^{3}$ and Naiqiang Cui ${ }^{4}$ \\ ${ }^{1}$ Department of Gastrointestinal Surgery, Nankai Hospital, Nankai District, Tianjin 300100, China \\ ${ }^{2}$ Graduate School of Tianjin Medical University, Nankai District, Tianjin 300100, China \\ ${ }^{3}$ Tianjin Institute of Acute Abdominal Disease of Integrated Traditional Chinese and Western Medicine, Nankai District, \\ Tianjin 300100, China \\ ${ }^{4}$ Department of Hepatopancreatobiliary Surgery, Nankai Hospital, Nankai District, Tianjin 300100, China
}

Correspondence should be addressed to Naiqiang Cui; nctsui@126.com

Received 17 September 2013; Revised 23 March 2014; Accepted 23 March 2014; Published 28 April 2014

Academic Editor: Sunil Kumar Khare

Copyright (C) 2014 Xiangyang Yu et al. This is an open access article distributed under the Creative Commons Attribution License, which permits unrestricted use, distribution, and reproduction in any medium, provided the original work is properly cited.

Intestinal obstruction is a common disease requiring abdominal surgery with significant morbidity and mortality. Currently, an effective medical treatment for obstruction, other than surgical resection or decompression, does not exist. Si-Jun-Zi Decoction is a famous Chinese medicine used to replenish qi and invigorate the functions of the spleen. Modern pharmacological studies show that this prescription can improve gastrointestinal function and strengthen immune function. In this study, we investigated the effects of a famous Chinese herbal formula, Si-Jun-Zi Decoction, on the restoration of intestinal function after the relief of obstruction in a rabbit model. We found that Si-Jun-Zi Decoction could reduce intestinal mucosal injury while promoting the recovery of the small intestine. Further, Si-Jun-Zi Decoction could regulate the intestinal immune system. Our results suggest that Si-Jun-Zi Decoction promotes the restoration of intestinal function after obstruction by regulating intestinal homeostasis. Our observations indicate that Si-Jun-Zi Decoction is potentially a therapeutic drug for intestinal obstruction.

\section{Introduction}

Intestinal obstruction is a common disease requiring abdominal surgery with significant morbidity and mortality. Intestinal obstruction often occurs in the small or large intestines [1]. Regardless of the initial cause of the obstruction, a series of pathophysiological changes occur in the obstructed segments. These changes are responsible for symptoms such as bloating, vomiting, abdominal cramps, and constipation and may lead to intestinal failure [2].

The function of the intestine rests on the normal and balanced homeostasis of the intestine, while intestinal homeostasis depends upon complex interactions between the intestinal epithelium and the intestinal immune system [3]. On a cellular level, the dynamic crosstalk between intestinal epithelial cells (IECs) and local immune cells represents one of the fundamental features of intestinal homeostasis. These interactions are not only important for the pathogenesis of intestinal disorders such as IBD, Crohn's disease, and intestinal obstruction, but also essential for maintaining normal intestinal homeostasis [4]. Our previous study has shown that intestinal obstruction can induce severe dysfunction of intestinal homeostasis. The intestinal epithelial cells and the intestinal immune system are significantly compromised as the obstruction progresses. Therefore, restoration of intestinal homeostasis may be an attractive strategy for treatment of human intestinal obstruction.

The Chinese medicine Si-Jun-Zi Decoction is a famous herbal formula composed of four Chinese herbs: Ginseng Root, Atractylodes macrocephala, licorice root, and Poria root. 
This formula is considered mild in nature. It balances qi and invigorates the spleen. Modern pharmacological studies show that this prescription affects several other physiological functions such as improving gastrointestinal function [5], strengthening the immune system $[6,7]$, improving bone marrow hematopoietic function, and speeding up the production of red blood cells. This prescription can also be used for the treatment of malignant tumors of the digestive tract [8]. Xiao and Yang found that Si-Jun$\mathrm{Zi}$ Decoction could improve the immune function and quality of life and reduce the side effects of chemotherapy in patients with colorectal cancer undergoing chemotherapy [7]. Liu et al. found that Si-Jun-Zi Decoction could promote the recovery of glucose uptake in the small intestine of reserpine induced Pi-qi deficiency syndrome rats [9]. Modified Si-Jun-Zi Decoction could slow down the formation of prednisone-induced osteoporosis through promoting osteoblast differentiation and inhibiting osteoclastogenesis [10]. A clinical observation by Guo et al. found that Chenxia Si-Jun-Zi Decoction could promote severe patient's gastrointestinal function recovery and reduce hospitalization days [5]. However, the therapeutic effects of Si-Jun-Zi Decoction on cases of intestinal obstruction are unknown.

In the present study, we investigate the effects of $\mathrm{Si}$ Jun-Zi Decoction on the restoration of intestinal function after the relief of obstruction in a rabbit model. Our results indicate that Si-Jun-Zi Decoction promotes the restoration of intestinal function by regulating intestinal homeostasis. Our observations indicate that Si-Jun-Zi Decoction may have potential therapeutic effects on patients suffering from intestinal obstruction.

\section{Materials and Methods}

2.1. Animals and Reagents. Healthy New Zealand rabbits with a body weight of $2.5-3 \mathrm{~kg}$ were used in this study. The animals were purchased from Mingle Laboratory Animal Center (Tianjin, China) and maintained in a temperaturecontrolled room with a $12 \mathrm{~h} \mathrm{light/dark}$ cycle and with access to regular chow and water. The experimental procedures were approved by the Laboratory Animal Care Committee at Tianjin Medical University. All animals received care according to the Guide for the Care and Use of Laboratory Animals (NIH, Bethesda, MD). The Si-Jun-Zi Decoction was supplied by the pharmaceutical preparation section of Nankai Hospital (Tianjin, China). The formula was composed of tuckahoe root (Scierotium poriae cocos), ginseng root (Radix ginseng), white Atractylodes rhizome root (Rhizoma Atractylodes macrocephalae), and licorice root (Radix glycyrrhizae) in the ratio of $3: 3: 2: 2$ (tuckahoe root $60 \mathrm{~g}$, ginseng root $60 \mathrm{~g}$, rhizome root $60 \mathrm{~g}$, and licorice root $40 \mathrm{~g}$ ), which were submerged in $2.2 \mathrm{~L}$ distilled water for $30 \mathrm{~min}$ and then decocted twice ( $2 \mathrm{~h}$ per time), then filtered and concentrated to $1 \mathrm{~g} / \mathrm{mL}$ using a routine method, and stored in a refrigerator at $4^{\circ} \mathrm{C}$ until use.
2.2. Description of Experimental Groups and the Rabbit Intestinal Obstruction Model. Thirty-six New Zealand rabbits were randomly divided into six groups: sham operation control group (Sham), $48 \mathrm{~h}$ after obstruction group $\left(\mathrm{O}_{48 \mathrm{~h}}\right)$, natural recovery at $48 \mathrm{~h}$ or $96 \mathrm{~h}$ after relief of the obstruction groups $\left(\mathrm{S}_{48 \mathrm{~h}}, \mathrm{~S}_{96 \mathrm{~h}}\right)$, and Si-Jun-Zi Decoction treatment for $48 \mathrm{~h}$ or $96 \mathrm{~h}$ after the relief of the obstruction groups $\left(\mathrm{T}_{48 \mathrm{~h}}\right.$, $\left.\mathrm{T}_{96 \mathrm{~h}}\right),(n=6$ per group $)$. To establish a rabbit model of intestinal obstruction that was controllable, we transformed the parts of infusion sets that are widely used in clinical applications into an in vitro pulled-type lock. After being anesthetized with an i.v. injection of urethane $(1 \mathrm{~g} / \mathrm{kg})$, a laparotomy was performed on sedated rabbits and a uniform controllable loop obstruction was created in the mesenteric nonavascular zone by placing a clamp eight $\mathrm{cm}$ from the distal end of the ileum. Sham-operated rabbits received mock manipulation of the gut without placement of the lock. The animals were allowed to recover postoperatively for 3 days to remove any influence of the anesthesia on our test parameters. Three days after the operation, the clamp was locked according to the color label, resulting in the obstruction of the intestine. The obstruction lasted for $48 \mathrm{~h}$ after which the intestinal obstruction was relieved by cutting off the lock. The animals were then treated with or without drug $(5 \mathrm{~g} / \mathrm{kg}$, twice a day) for $48 \mathrm{~h}$ or $96 \mathrm{~h}$. At the end of the experiment, the animals were sacrificed for further analysis.

\subsection{Quantification of Intestinal Damage and Determina-} tion of Intraepithelial Lymphocytes (IEL) and Lamina Propria Lymphocytes (LPL) Cell Numbers. Two-centimeter or longer intestinal segments from the ileum $(5 \mathrm{~cm}$ from the distal ileum with obstruction) were excised, fixed in $4 \%$ paraformaldehyde, and embedded in paraffin blocks. Fourmicron thick sections were stained with $\mathrm{H} \& \mathrm{E}$ and PAS using standard protocols [11]. The sections were examined using a Champion-500w graphic report management system. Intestinal mucous membrane damage was evaluated and Chiu's [12] histopathological scores were determined. Briefly, the tissue damage was graded from 0 to 5 according to the following criteria: grade 0 , normal structure of villi; grade 1 , development of small subepithelial space at the villous apex; grade 2, enlarged subepithelial space but without change in villous length and width; grade 3 , few shortened villi and presence of cells in the lumen; grade 4, the majority of villi are shortened and widened with crypt hyperplasia and cells in the lumen; and grade 5, blunting of all villi with elongated crypts and a large number of cells in the lumen. The percentage of intraepithelial lymphocytes (IELs) and lamina propria lymphocytes (LPLs) were quantified in photomicrographs of the intestines. The percentage of IEL cells was calculated using the following formula: (the number of total IEL cells in 20 villi per section/the total cell number in 25 villi per section) $\times$ 100. The percentage of LPL cells was calculated using the following formula: (the number of total LPL cells in 20 villi per section/the total lamina propria cell number in the central axis of 25 villi per section) $\times 100$. 
2.4. High-Performance Liquid Chromatography (HPLC) Analysis of Ornithine Decarboxylase Activity and Citrulline Levels. Theornithine decarboxylase (ODC) activity of intestinal tissues and the level of citrulline in blood serum were analyzed by HPLC as described elsewhere $[13,14]$.

2.5. RNA Extraction and Real-Time PCR. RNA was extracted from intestinal segments $(100 \mathrm{mg})$ from the ileum $(5 \mathrm{~cm}$ from the distal ileum with obstruction) using a Total RNA Kit (Qiagen) by following the manufacturer's instructions. First-strand cDNA was synthesized from $1 \mu \mathrm{g}$ mRNA using reverse transcriptase (Fermentas, Glen Burnie, MD) and oligo (dT) primers. The primer sequences used were as follows: Claudin 1 forward: $5^{\prime}$-GTGCCTTGATGGTGATTG- ${ }^{\prime}$, reverse: $5^{\prime}$-AAAGTAGCCAGACCTGAAAT- $3^{\prime}$. $\beta$-actin forward: $5^{\prime}$-TGATGGTGGGCATGGGTC- ${ }^{\prime}$, reverse: $5^{\prime}$-CGATGGGGTACTTCAGGGTG-3' ${ }^{\prime}$. Real-time PCR was performed using an Applied Biosystems PRISM7300 (Applied Biosystems) and SYBR Green PCR master mix (Applied Biosystems). Reagents concentration were $2 \times$ Realtime Mix $10 \mu \mathrm{L}$, forward primer $0.3 \mu \mathrm{L}$, reverse primer $0.3 \mu \mathrm{L}, \mathrm{dd}_{2} \mathrm{O}$ $7.4 \mu \mathrm{L}$, and cDNA templates $2 \mu \mathrm{L}$. PCR cycling conditions were $95^{\circ} \mathrm{C}$ for $30 \mathrm{sec}$ followed by 40 cycles of $95^{\circ} \mathrm{C}$ for $5 \mathrm{sec}$ and $50^{\circ} \mathrm{C}$ for $30 \mathrm{sec}$. The mRNA expression was normalized to the expression of the $\beta$-actin housekeeping gene.

2.6. Flow Cytometry Analysis of Peyer's Patch (PP) Lymphocytes. The PP lymphocytes were isolated as described previously [15]. Briefly, the excised PP lymphocytes were incubated under sterile conditions with RPMI medium containing $1 \mathrm{mM}$ DTT $\left(5 \mathrm{~min}, 37^{\circ} \mathrm{C}\right)$. Thereafter, the PP lymphocytes were washed with RPMI medium and passed through a steel mesh. The resulting cell suspension was washed and resuspended in RPMI containing 10\% FBS. The PP lymphocytes were stained with FITC-anti-rabbit CD4, PE-anti-rabbit CD8, and Percp-Cy5.5-anti-rabbit CD3. A negative control was stained with isotype-matched $\mathrm{mAb}$. Cells were stained at $4^{\circ} \mathrm{C}$ for $30 \mathrm{~min}$. The cells were then washed and resuspended in PBS for FACS analysis. Data were acquired using a FACSCalibur (BD Bioscience) flow cytometer and analyzed using the CellQuest software.

2.7. ELISA. A fresh $8 \mathrm{~cm}$ intestinal segment from the ileum $(5 \mathrm{~cm}$ from the distal ileum with obstruction) was obtained, the intestinal solid contents were removed, and casing slime was collected by douching with $3 \mathrm{~mL}$ of sterile PBS. The obtained extracts were centrifuged and the supernatants were collected. The levels of s-IgA were measured using an ELISA kit according to the manufacturer's instructions. The serum level of D-lactate was measured by ELISA kit (ADL co.), according to the manufacturer's instructions.

2.8. Statistical Analysis. Results were expressed as mean \pm $S D$. The data were analyzed using a two-tailed Student's $t$-test
(GraphPad Prism 5) and a $P$ value of $<0.05$ was regarded as statistically significant.

\section{Results}

3.1. The Si-Jun-Zi Decoction Reduces Intestinal Mucosal Injury. The intestinal mucosal barrier is the first line of host defense against intestinal pathogens, and intestinal obstruction induces sever intestinal mucosal injury [16]. Intestinal mucosal injury is also a common clinical complication that may lead to dysfunction of the intestinal barrier. Here, by using a modified and controllable rabbit intestinal obstruction model, we evaluated the ability of the Si-Jun$\mathrm{Zi}$ Decoction to reduce the obstruction-induced intestinal mucosal damage after relief of the intestinal obstruction. As shown in Figures 1(a) and 1(b), the intestinal obstruction induced severe intestinal mucosal injury, including villous blunting and epithelial sloughing. The overall histopathological damage score of the intestines of the sham and $\mathrm{O}_{48} \mathrm{~h}$ groups was statistically different. After relieving the obstruction, the damage to the intestinal mucosa was gradually repaired, while the Si-Jun-Zi Decoction treatment groups showed significantly increased levels of repair compared to treatment groups without Si-Jun-Zi Decoction treatment. The overall histopathological damage score of the $\mathrm{S}_{48 \mathrm{~h}}$ versus $T_{48} \mathrm{~h}$ groups and the $S_{96}$ versus $T_{96}$ groups was statistically different $(P<0.05)$, suggesting that the SiJun-Zi Decoction treatment had a profound protective effect against intestinal mucosal injury. This protection provided by the Si-Jun-Zi Decoction was confirmed by changes in the level of blood D-lactate, which is a measure of intestinal mucosa permeability [17], and the intestinal mucins, which are key components of the intestinal mucosal barrier [18]. The data showed that $\mathrm{D}$-lactate levels significantly declined after Si-Jun-Zi Decoction treatment $\left(\mathrm{S}_{48 \mathrm{~h}}\right.$ versus $\mathrm{T}_{48 \mathrm{~h}}$ and $S_{96 \mathrm{~h}}$ versus $\mathrm{T}_{96 \mathrm{~h}}$ ) (Figure 1(c)), while levels of the intestinal mucins significantly increased relative to the control groups $\left(\mathrm{S}_{48 \mathrm{~h}}\right.$ versus $\mathrm{T}_{48 \mathrm{~h}}$ and $\mathrm{S}_{96 \mathrm{~h}}$ versus $\mathrm{T}_{96 \mathrm{~h}}$ ) (Figure $1(\mathrm{~d})$ ). These results indicate that the Si-Jun-Zi Decoction could reduce the permeability of intestinal mucosa and enhance the secretion of intestinal mucins. Taken together, our results showed that the Si-Jun-Zi Decoction was beneficial for the reduction of intestinal mucosal injury after relieving the intestinal obstruction.

3.2. The Si-Jun-Zi Decoction Promotes the Recovery of the Small Intestine. We next evaluated the effects of the Si-Jun-Zi Decoction on the function and integrity of the small intestine. We first examined the change in ornithine decarboxylase (ODC) levels, which indicate intestinal epithelial cells proliferation [19]. Our previous study showed that the level of ODC increased after obstruction, reaching a peak at $12 \mathrm{~h}$ after obstruction, and then rapidly decreased. This observation suggests that, during the early stages of obstruction, the intestinal epithelial cells renewed quickly, promoting the recovery of the intestinal epithelium, and that the damage and recovery were balanced. However, at later stages, this balance was upset due to the severity of intestinal epithelial damage. 

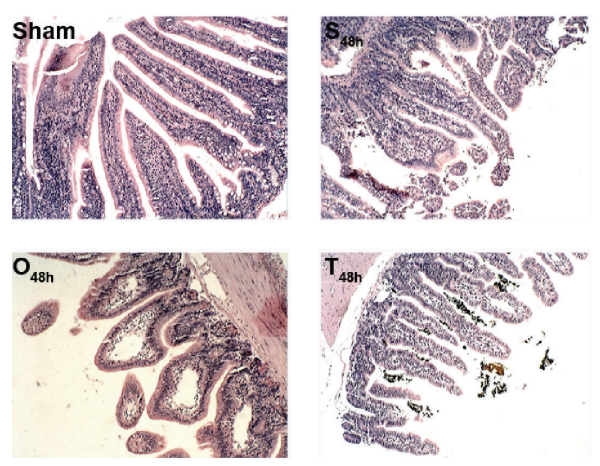

(a)

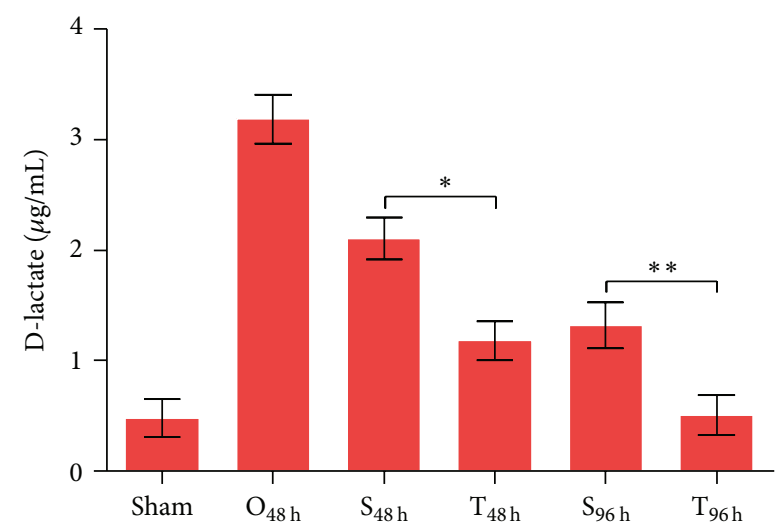

(c)
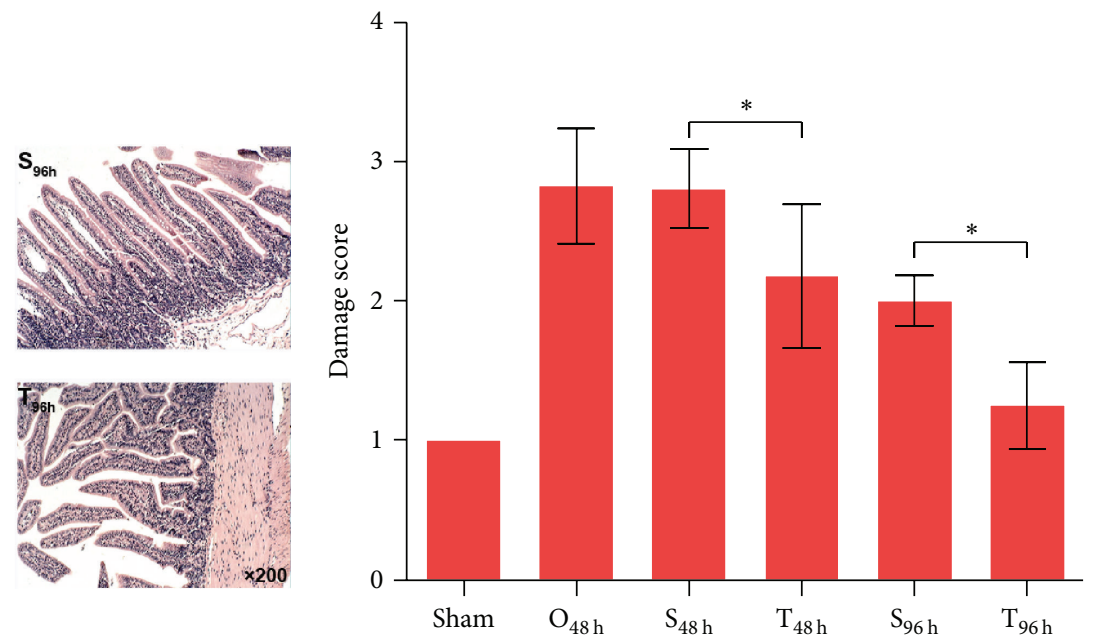

(b)

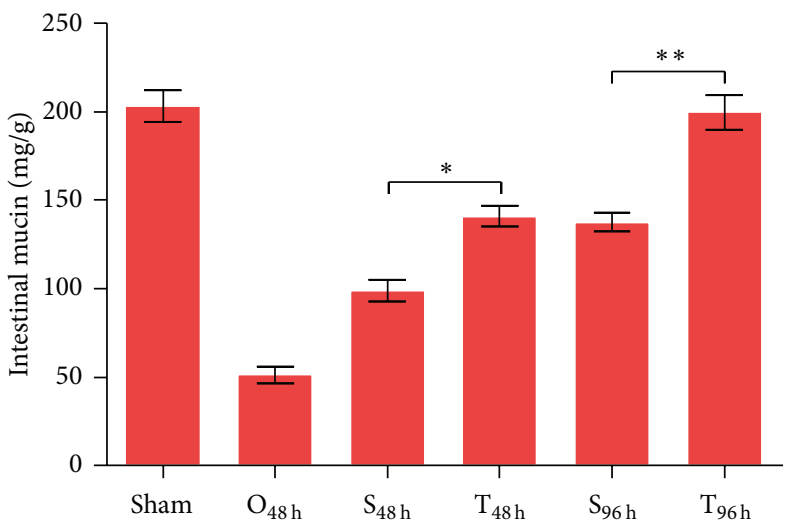

(d)

FIGURE 1: Si-Jun-Zi Decoction treatment reduces intestinal mucosal injury. (a) and (b): intestinal segments from the ileum were excised and fixed in $4 \%$ paraformaldehyde and embedded in paraffin blocks. We stained $4 \mu \mathrm{m}$ thick sections with H\&E. Intestinal mucous membrane damage and Chiu's histopathological score were evaluated. The H\&E staining (a) and the damage score (b) are shown. In panel (c), the serum level of D-lactate is shown. In panel (d), the level of intestinal mucins, examined by Coomassie brilliant blue G250 staining, is shown. The data are representative of three independent experiments, with each using 6 mice per group. Data are shown as mean \pm SD. $\left({ }^{*} P<0.05\right.$, $\left.{ }^{* *} P<0.01\right)$.

As shown in Figure 2(a), the ODC level of the $\mathrm{O}_{48} \mathrm{~h}$ group was equal to the sham control group and gradually increased after the obstruction was relieved. The level of ODC was significantly elevated after $48 \mathrm{~h}$ of Si-Jun-Zi Decoction compared with the $\mathrm{S}_{48} \mathrm{~h}$ group. At $96 \mathrm{~h}$ after relieving the obstruction, the ODC level returned to normal and thus maintained intestinal homeostasis. This result indicates that the Si-Jun$\mathrm{Zi}$ Decoction could promote the recovery of the intestinal epithelium. We next quantified the level of blood citrulline, which serves as an indicator of the absorptive function of small intestine [20]. Our data showed that serum levels of citrulline in the $\mathrm{T}_{48 \mathrm{~h}}$ or $\mathrm{T}_{96 \mathrm{~h}}$ groups were significantly higher than those in the $S_{48 \mathrm{~h}}$ or $S_{96 \mathrm{~h}}$ groups (Figure 2(b)), suggesting that the Si-Jun-Zi Decoction treatment promoted the recovery of the absorptive function of the small intestine. Because the integrity of the intestinal mucosa, which is maintained by tight junction proteins (TJPs) and adherens junction proteins, is essential for the function of the intestinal barrier [21], we examined Claudin 1 gene expression by real-time PCR analysis. Claudin 1 is the functional component of tight junction transmembrane protein (TJPs) in intestinal epithelial cells [22]. We found almost undetectable levels of Claudin 1 gene expression at $48 \mathrm{~h}$ after obstruction, indicating that the intestinal epithelial integrity at later stages of obstruction was completely lost (Figure 2(c)). Once the obstruction was removed, Claudin 1 gene expression increased ( $\mathrm{S}_{48 \mathrm{~h}}$ group). In animals treated with the Si-Jun-Zi Decoction for $48 \mathrm{~h}$, expression of the Claudin 1 gene was significantly increased compared with the $S_{48}$ group, and this enhancement was more obvious in $\mathrm{T}_{96 \mathrm{~h}}$ group versus $\mathrm{T}_{48 \mathrm{~h}}$ group. This result 


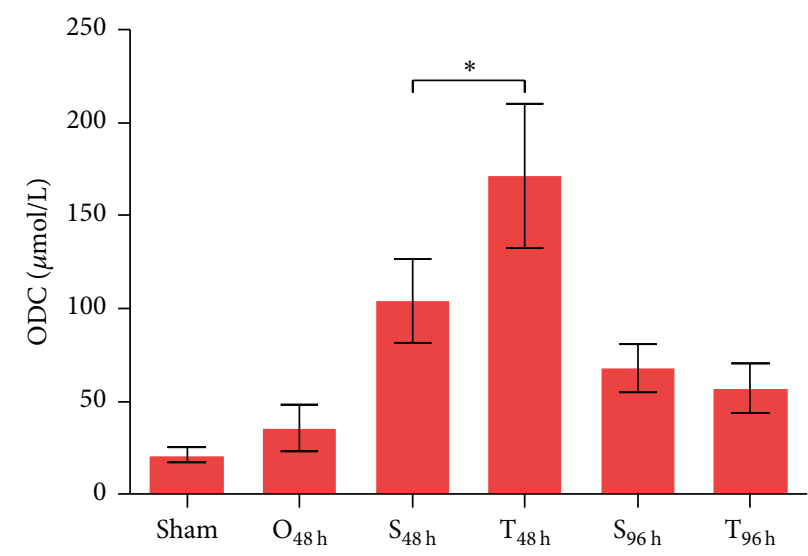

(a)

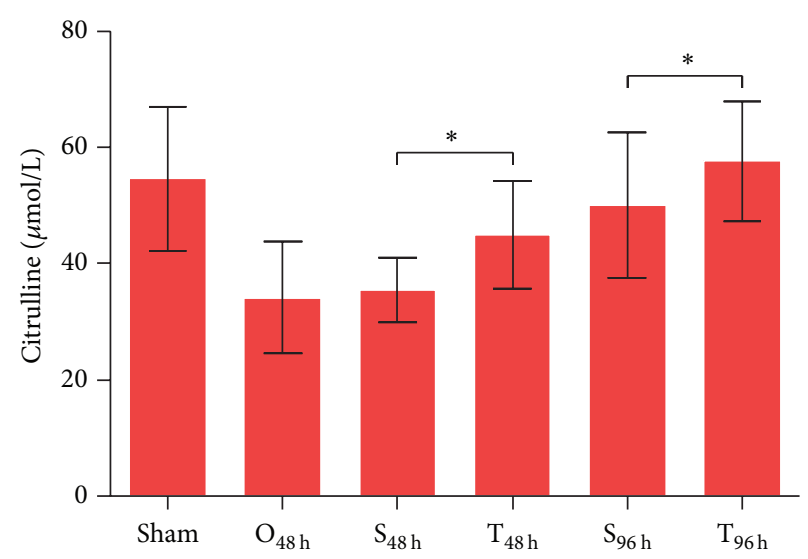

(b)

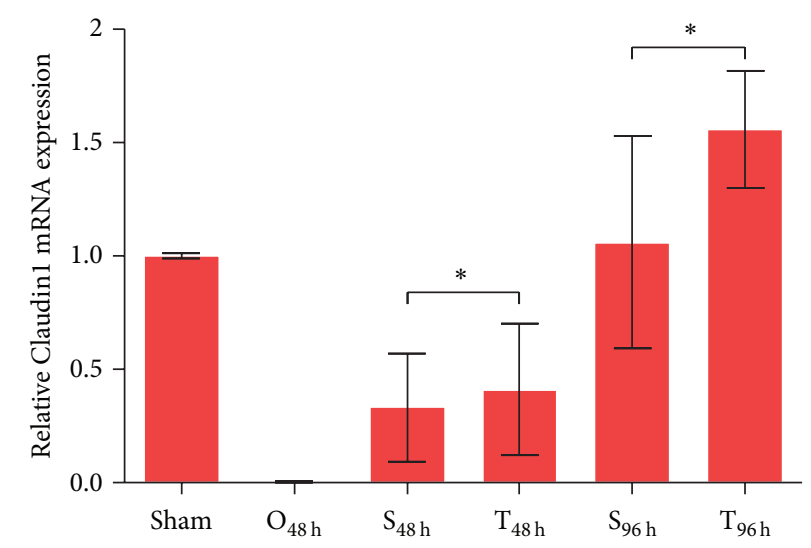

(c)

FIGURE 2: Si-Jun-Zi Decoction treatment promotes recovery of the small intestine. (a) and (b): the ODC activity (a) in intestinal tissues and the level of citrulline (b) in blood serum were analyzed by HPLC. The data are shown as mean $\pm \operatorname{SD}(n=6)$. (c) The gene expression level of Claudin 1 was examined by qRT-PCR. The data are shown as the mean \pm SD $(n=6)$. The data are representative of at least three independent experiments. ${ }^{*} P<0.05$.

indicates that the Si-Jun-Zi Decoction could promote the recovery of the integrity of the small intestine. Taken together, our results suggest that the Si-Jun-Zi Decoction can promote the recovery of the small intestine after relieving intestinal obstruction.

\subsection{The Si-Jun-Zi Decoction Regulates the Intestinal Immune} System. Intestinal epithelial cells are well known for their role as the boundary between the external environment and the intestinal tract. Apart from being a physical barrier, intestinal epithelial cells play a pivotal role in regulating immune responses in order to maintain intestinal homeostasis [23]. However, once intestinal epithelial cell damage occurs, the balance between intestinal epithelial cell barrier and intestinal immune system cannot be maintained. Our previous study found that intestinal obstruction induces intestinal immune system dysfunction and therefore we investigated whether the Si-Jun-Zi Decoction could regulate the intestinal immune system after intestinal obstruction was relieved. We first assessed the number of intraepithelial lymphocytes (IELs) and lamina propria lymphocytes (LPLs) and intestinal epithelial cells (IEC) using PAS stained tissue sections. The data showed that significant differences in the numbers of IELs after Si-Jun-Zi Decoction treatment (data not shown) were not found, while the number of LPLs decreased with the Si-Jun-Zi Decoction treatment $\left(S_{48} \mathrm{~h}\right.$ versus $\mathrm{T}_{48 \mathrm{~h}}, P<0.05$ ) (Figure 3(a)). In addition, we analyzed the level of s-IgA in the intestinal lumen at various times, with or without drug treatment, after relieving the obstruction. We found that the s-IgA level was not significantly changed, suggesting that the Si-Jun-Zi Decoction does not affect the innate immunity of the intestine. We next examined alterations in $\mathrm{T}$ cells subtype percentages in PP lymphocytes by flow cytometry. As shown in Figures 3(c)-3(f), Si-Jun$\mathrm{Zi}$ Decoction treatment could reduce the percentage of $\mathrm{CD}^{+} \mathrm{T}$ cells $48 \mathrm{~h}$ after relieving the intestinal obstruction compared with the no treatment group (Figure 3(c)). SiJun- $\mathrm{Zi}$ Decoction treatment could increase the percentage of $\mathrm{CD} 4^{+} \mathrm{T}$ cells and reduce the percentage of $\mathrm{CD} 8^{+} \mathrm{T}$ cells at 


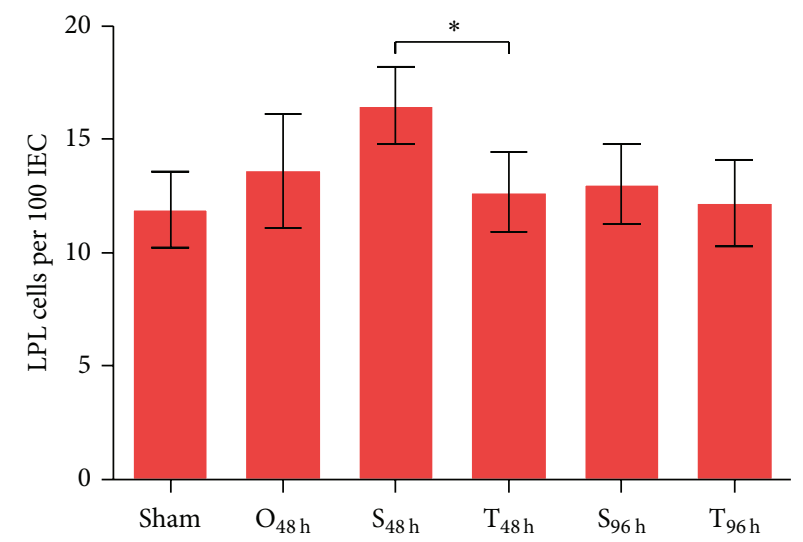

(a)

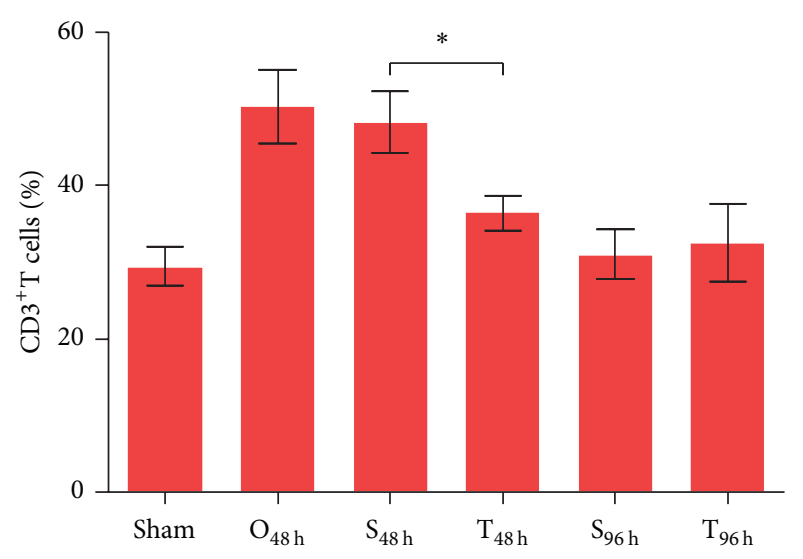

(c)

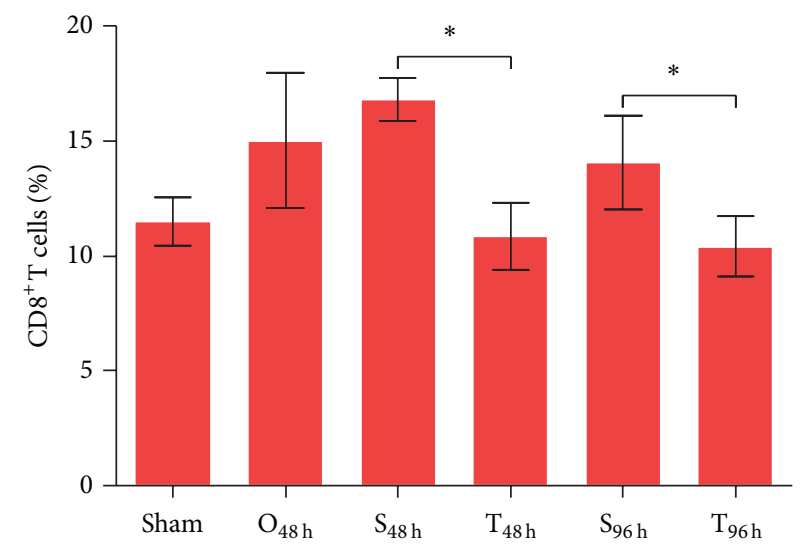

(e)

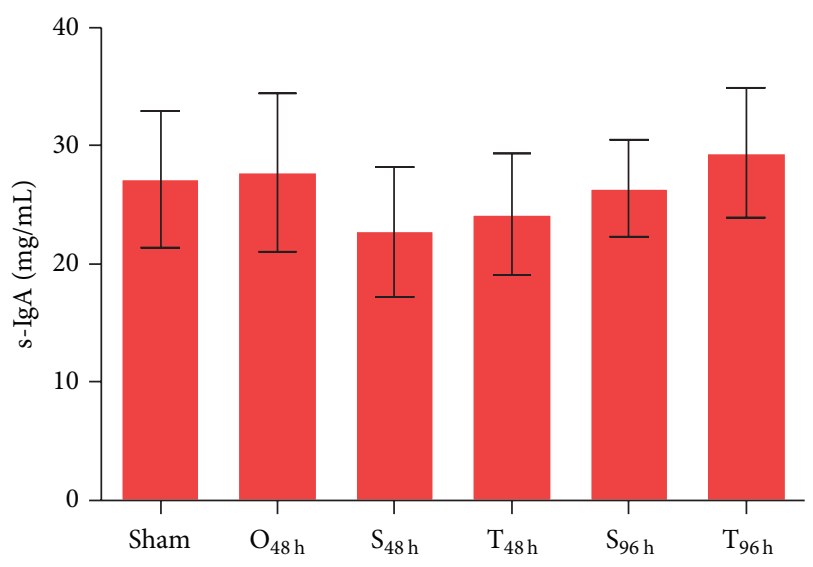

(b)

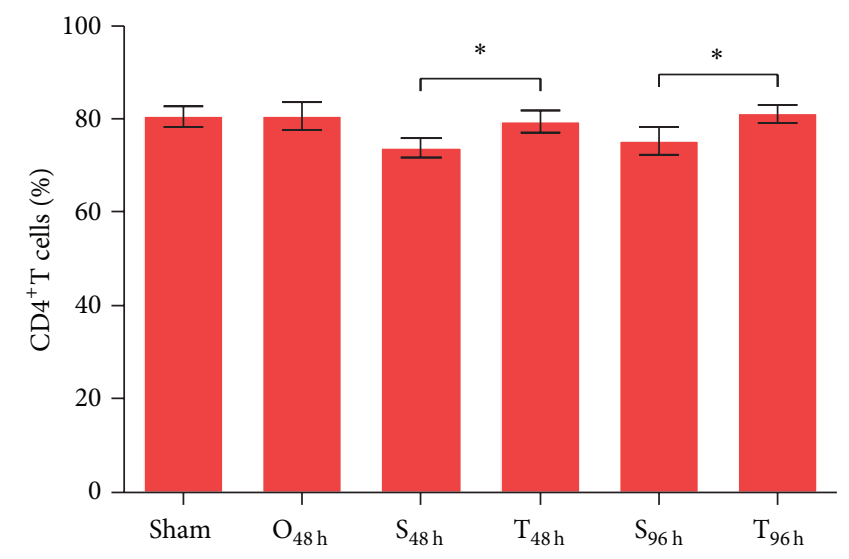

(d)

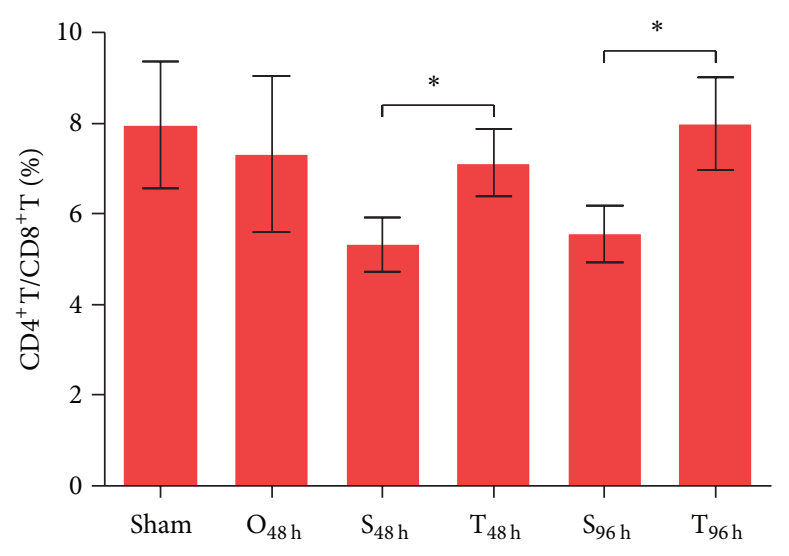

(f)

FIGURE 3: Si-Jun-Zi Decoction treatment regulates the intestinal immune system. (a) Intestinal segments from the ileum were excised and fixed in $4 \%$ paraformaldehyde and embedded in paraffin blocks. We stained $4 \mu \mathrm{m}$ thick sections with PAS, and the percentage of LPLs was quantified in photomicrographs of the intestines. (b) The level of s-IgA in the intestinal lumen was examined by ELISA. (c)-(f) The PP lymphocytes were isolated and the percentage of $\mathrm{T}$ cells subtypes was analyzed by flow cytometry. The percentage of $\mathrm{CD} 3^{+} \mathrm{T}$ cells $(\mathrm{c})$, the percentage of $\mathrm{CD} 4^{+} \mathrm{T}$ cells $(\mathrm{d})$, the percentage of $\mathrm{CD} 8^{+} \mathrm{T}$ cells $(\mathrm{e})$, and the $\mathrm{CD} 4^{+} / \mathrm{CD} 8^{+} \mathrm{T}$ cells ratio (f) are shown. The data are representative of three independent experiments, with each using 4-5 mice per group. Data are shown as mean $\pm \operatorname{SD}\left({ }^{*} P<0.05\right)$.

different times with or without drug treatment after the relief of obstruction (Figures $3(\mathrm{~d})$ and $3(\mathrm{e})$ ). The $\mathrm{CD} 4^{+} / \mathrm{CD} 8^{+} \mathrm{T}$ cells ratio was also sharply elevated by Si-Jun-Zi Decoction treatment (Figure 3(f)). These data suggest that the Si-Jun-Zi
Decoction could regulate the adaptive immune response by reducing the number of $\mathrm{CD}^{+} \mathrm{T}$ cells and $\mathrm{CD} 8^{+} \mathrm{T}$ cells while increasing the number of $\mathrm{CD} 4^{+} \mathrm{T}$ cells. However, the precise mechanisms involved still require further investigation. 
Based on the above data, we suggest that the Si-Jun-Zi Decoction can promote the restoration of intestinal function by regulating the intestinal immune system.

\section{Discussion}

Intestinal obstruction is a common disease requiring abdominal surgery with significant morbidity and mortality [1]. The major morbidity associated with intestinal obstruction is related to excessive distension that leads to strangulation and bowel necrosis. Until now, effective medical treatment for obstruction, other than surgical resection or decompression, is lacking. However, even if the obstruction is surgically removed, many patients continue to have disturbed motility function in the bowel proximal to the site of resection for many years to come $[24,25]$. There are very few drugs for the treatment of intestinal obstructions. Recently Alvimopan, a drug that behaves as a peripherally acting $\mu$-opioid receptor antagonist, was approved by the FDA for the treatment of patients with postoperative ileus (POI) following partial large or small bowel resection surgery with primary anastomosis [26]. However, there are several side effects and limitations for the use of this drug $[27,28]$.

Si-Jun-Zi Decoction is a famous Chinese medicine used to replenish qi and invigorate the functions of the spleen. It is used when there is a deficiency of qi of the spleen and stomach, which is marked by anorexia and loose bowels. Modern pharmacological studies show that this prescription can improve gastrointestinal function [5] and strengthen immune function $[6,7]$. Therefore, we hypothesized that this prescription may be beneficial for the restoration of intestinal function after relieving the obstruction.

In the current study, we used a controllable rabbit intestinal obstruction model to investigate the effects of a SiJun-Zi Decoction on the restoration of intestinal function after relieving the obstruction. We found that the Si-Jun$\mathrm{Zi}$ Decoction can reduce the intestinal mucosal injury. SiJun-Zi Decoction treatment significantly alleviated intestinal mucosal damage, reduced intestinal mucosal permeability, and enhanced the secretion of intestinal mucins. Furthermore, the Si-Jun-Zi Decoction promotes the recovery of the small intestines. Si-Jun-Zi Decoction treatment could promote the recovery of both the intestinal epithelium and the absorptive function of the small intestine by increasing ODC and citrulline levels. In addition, the Si-Jun-Zi Decoction promotes the recovery of small intestine integrity by upregulating the Claudin 1 gene expression. Finally, we found that the Si-Jun-Zi Decoction regulates the intestinal immune system. Although the Si-Jun-Zi Decoction does not affect the innate immunity of the intestine, it may regulate the adaptive immune response by reducing $\mathrm{CD}^{+} \mathrm{T}$ cell and $\mathrm{CD} 8^{+} \mathrm{T}$ cell numbers while increasing the number of $\mathrm{CD} 4^{+} \mathrm{T}$ cells in $\mathrm{PP}$ lymphocytes.

Although we found that the famous Chinese herbal formula Si-Jun-Zi Decoction has the ability to promote the restoration of intestinal function by regulating the intestinal homeostasis, the precise mechanisms involved are still to be elucidated. Si-Jun-Zi Decoction as a potential therapeutic drug for intestinal obstruction treatment needs to be investigated in the future.

\section{Conclusion}

The present study clearly demonstrates that Si-Jun-Zi Decoction promotes the restoration of intestinal function after obstruction by regulating intestinal homeostasis. Our results indicate that the Si-Jun-Zi Decoction may be a potential therapeutic drug for intestinal obstruction. Further studies are needed to assess the side effects and limitations of this drug in the treatment of intestinal obstruction.

\section{Conflict of Interests}

The authors declare that there is no conflict of interests regarding the publication of this paper.

\section{Authors' Contribution}

Xiangyang Yu and Zhigang Cui contributed equally to this work.

\section{Acknowledgments}

This work was supported by the National Program on Key Basic Research Project (Grant no. 2009CB522703).

\section{References}

[1] M. S. Cappell and M. Batke, "Mechanical obstruction of the small bowel and colon," Medical Clinics of North America, vol. 92, no. 3, pp. 575-597, 2008.

[2] M. D. Zielinski and M. P. Bannon, "Current management of small bowel obstruction," Advances in Surgery, vol. 45, no. 1, pp. $1-29,2011$.

[3] L. V. Hooper and A. J. MacPherson, "Immune adaptations that maintain homeostasis with the intestinal microbiota," Nature Reviews Immunology, vol. 10, no. 3, pp. 159-169, 2010.

[4] D. Artis, "Epithelial-cell recognition of commensal bacteria and maintenance of immune homeostasis in the gut," Nature Reviews Immunology, vol. 8, no. 6, pp. 411-420, 2008.

[5] J. H. Guo, G. Chen, S. Q. Yang, M. H. Wei, and X. Chen, "Clinical observation of the role of Chenxia Sijunzi decoction in promoting the recovery of gastrointestinal function in critically ill patients," Zhongguo Wei Zhong Bing Ji Jiu Yi Xue, vol. 24, no. 11, pp. 674-676, 2012.

[6] C. Liang, S.-H. Zhang, and Z.-D. Cai, "Effects of early intestinal application of sijunzi decoction on immune function in postoperational patients of gastrointestinal tumor," Zhongguo Zhong Xi Yi Jie He Za Zhi, vol. 25, no. 12, pp. 1070-1073, 2005.

[7] H. Xiao and J. Yang, "Immune enhancing effect of modified sijunzi decoction on patients with colorectal cancer undergoing chemotherapy," Zhongguo Zhong Xi Yi Jie He Za Zhi, vol. 31, no. 2, pp. 164-167, 2011.

[8] B. Wu and Z.-R. Xuan, "Progress in research on applying Sijunzi Decoction in treating digestive malignant tumor," Chinese Journal of Integrative Medicine, vol. 13, no. 2, pp. 156-159, 2007.

[9] J. Liu, W. F. Guo, L. Ren, and W. W. Chen, "Effect of sijunzi decoction on the intestinal glucose absorption in model rats of 
Pi-qi deficiency syndrome: an experimental research," Zhongguo Zhong Xi Yi Jie He Za Zhi, vol. 33, no. 10, pp. 1389-1393, 2013.

[10] J. Zheng, J. L. Liu, M. F. Lin et al., "Effect of modified sijunzi decoction on the bone metabolism of adriamycin induced nephropathy rats," Zhongguo Zhong Xi Yi Jie He Za Zhi, vol. 33, no. 10, pp. 1376-1381, 2013.

[11] J. R. McDole, L. W. Wheeler, K. G. McDonald et al., "Goblet cells deliver luminal antigen to $\mathrm{CD}_{103}{ }^{+}$dendritic cells in the small intestine," Nature, vol. 483, no. 7389, pp. 345-349, 2012.

[12] C. J. Chiu, A. H. McArdle, R. Brown, H. J. Scott, and F. N. Gurd, "Intestinal mucosal lesion in low-flow states. I. A morphological, hemodynamic, and metabolic reappraisal," Archives of Surgery, vol. 101, no. 4, pp. 478-483, 1970.

[13] M. I. Escribano and M. E. Legaz, "High performance liquid chromatography of the dansyl derivatives of putrescine, spermidine, and spermine," Plant Physiology, vol. 87, no. 2, pp. 519-522, 1988.

[14] C. Bai, C. C. Reilly, and B. W. Wood, "Identification and quantitation of asparagine and citrulline using high-performance liquid chromatography (HPLC)," Analytical Chemistry Insights, vol. 2, pp. 31-36, 2007.

[15] E. Ramiro-Puig, F. J. Pérez-Cano, S. Ramos-Romero et al., "Intestinal immune system of young rats influenced by cocoaenriched diet," The Journal of Nutritional Biochemistry, vol. 19, no. 8, pp. 555-565, 2008.

[16] T.-M. Chang, R.-H. Lu, and L.-M. Tsai, "Glutamine ameliorates mechanical obstruction-induced intestinal injury," Journal of Surgical Research, vol. 95, no. 2, pp. 133-140, 2001.

[17] V. L. Jørgensen and A. Perner, "Relationship between permeability and luminal concentrations of lactate and glycerol in the small intestine?" Intensive Care Medicine, vol. 30, no. 10, p. 1979, 2004.

[18] S. K. Linden, P. Sutton, N. G. Karlsson, V. Korolik, and M. A. McGuckin, "Mucins in the mucosal barrier to infection," Mucosal Immunology, vol. 1, no. 3, pp. 183-197, 2008.

[19] H. Pendeville, N. Carpino, J.-C. Marine et al., "The ornithine decarboxylase gene is essential for cell survival during early murine development," Molecular and Cellular Biology, vol. 21, no. 19, pp. 6549-6558, 2001.

[20] P. Crenn, C. Coudray-Lucas, L. Cynober, and B. Messing, "Post-absorptive plasma citrulline concentration: a marker of intestinal failure in humans," Transplantation Proceedings, vol. 30, no. 6, p. 2528, 1998.

[21] M. Bruewer, S. Samarin, and A. Nusrat, "Inflammatory bowel disease and the apical junctional complex," Annals of the New York Academy of Sciences, vol. 1072, pp. 242-252, 2006.

[22] D. Gunzel and M. Fromm, "Claudins and other tight junction proteins," Comprehensive Physiology, vol. 2, no. 3, pp. 1819-1852, 2012.

[23] J. Pott and M. Hornef, "Innate immune signalling at the intestinal epithelium in homeostasis and disease," EMBO Reports, vol. 13, no. 8, pp. 684-698, 2012.

[24] H.-Y. Kim, J.-H. Kim, S.-E. Jung, S.-C. Lee, K.-W. Park, and W.K. Kim, "Surgical treatment and prognosis of chronic intestinal pseudo-obstruction in children," Journal of Pediatric Surgery, vol. 40, no. 11, pp. 1753-1759, 2005.

[25] M. Menezes, M. Corbally, and P. Puri, "Long-term results of bowel function after treatment for Hirschsprung's disease: a 29year review," Pediatric Surgery International, vol. 22, no. 12, pp. 987-990, 2006.
[26] J. B. Leslie, "Alvimopan for the management of postoperative ileus," Annals of Pharmacotherapy, vol. 39, no. 9, pp. 1502-1510, 2005.

[27] P. Neary and C. P. Delaney, "Alvimopan," Expert Opinion on Investigational Drugs, vol. 14, no. 4, pp. 479-488, 2005.

[28] M. D. Crowell and J. K. DiBaise, "Is alvimopan a safe and effective treatment for postoperative ileus?" Nature Clinical Practice Gastroenterology \& Hepatology, vol. 4, no. 9, pp. 484485, 2007. 


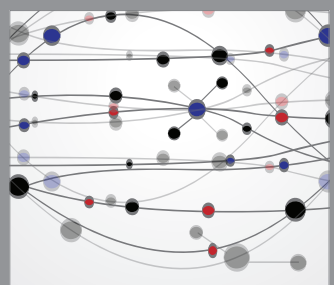

The Scientific World Journal
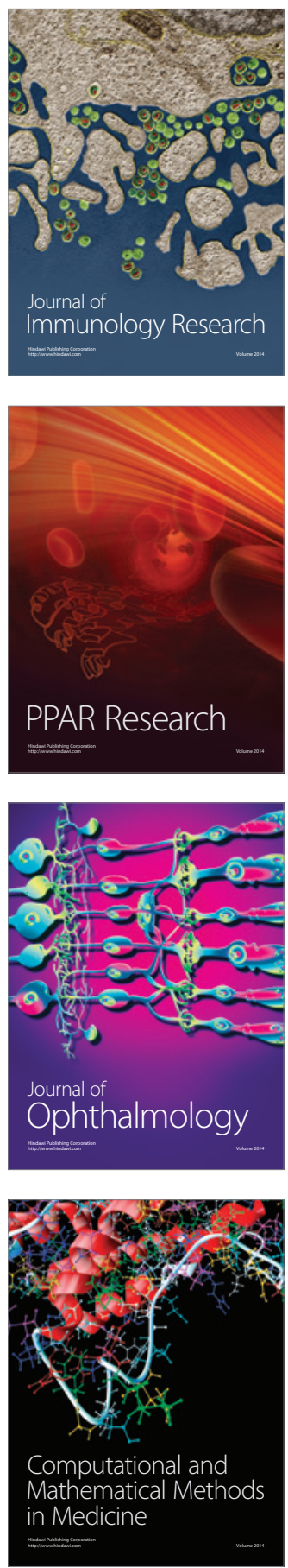

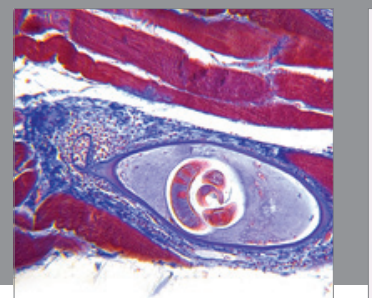

Gastroenterology

Research and Practice
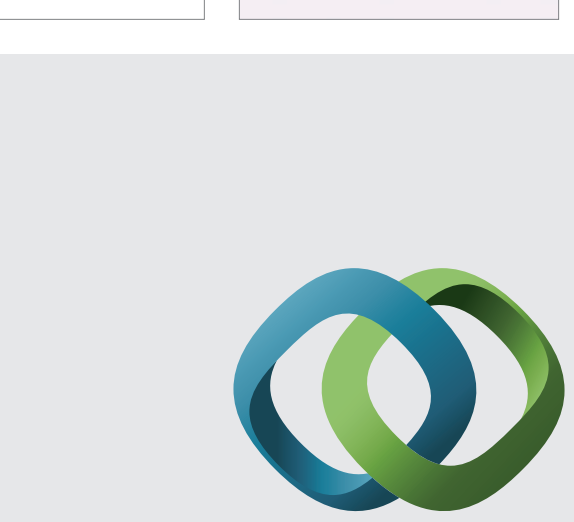

\section{Hindawi}

Submit your manuscripts at

http://www.hindawi.com
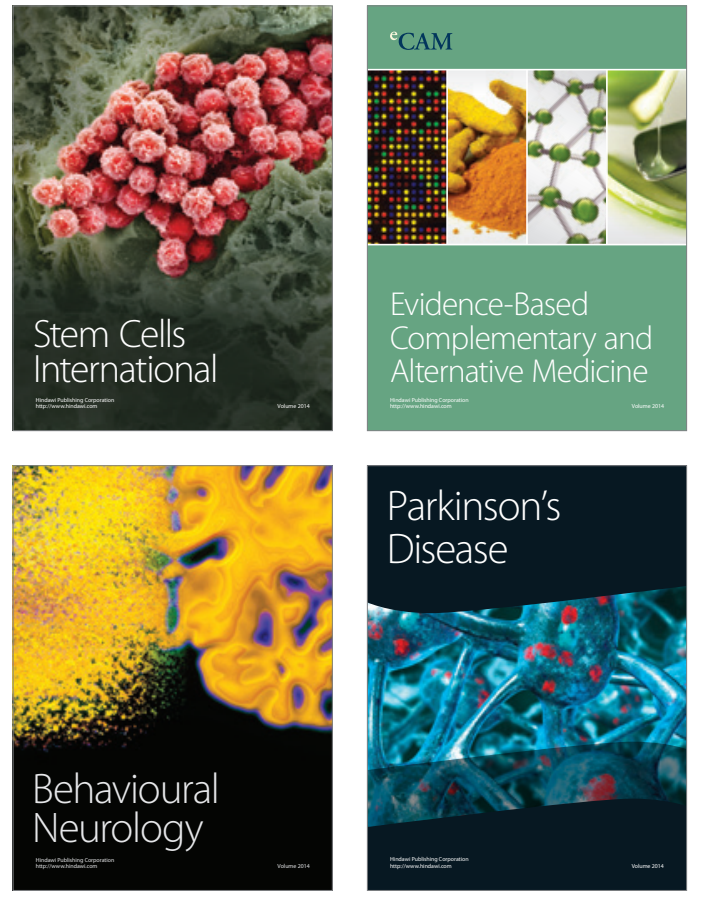
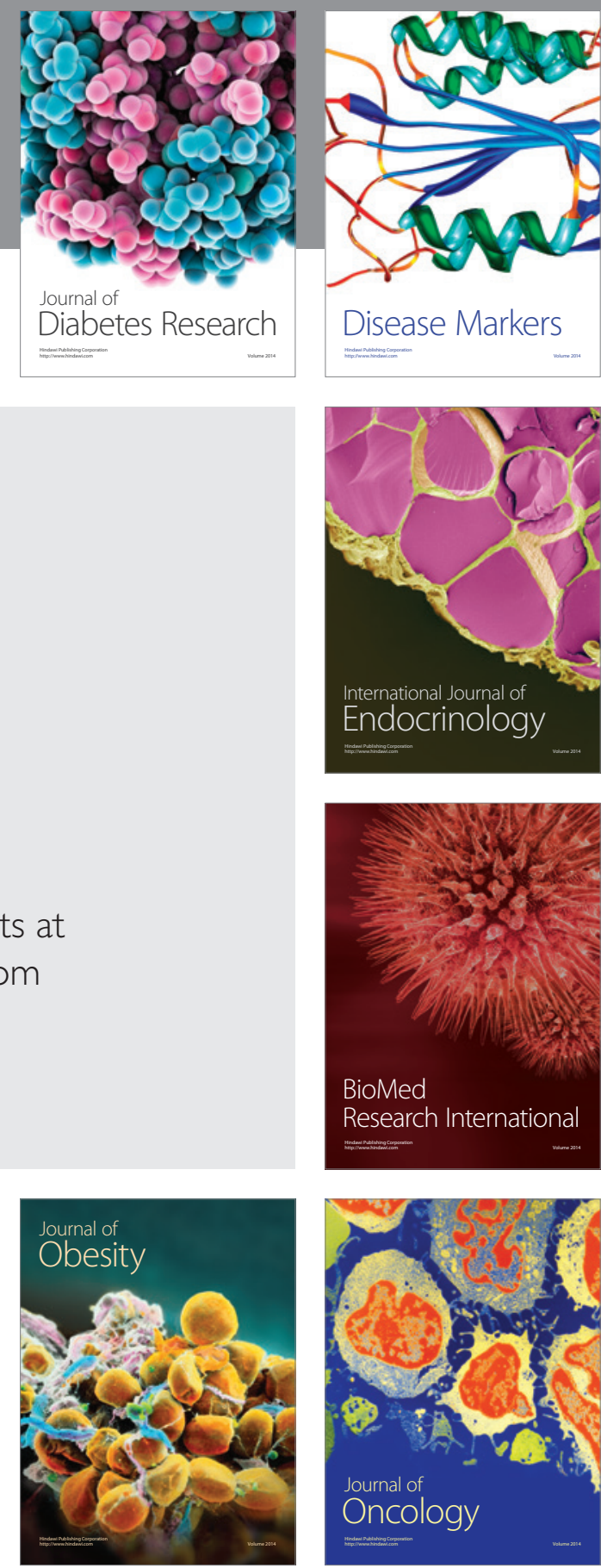

Disease Markers
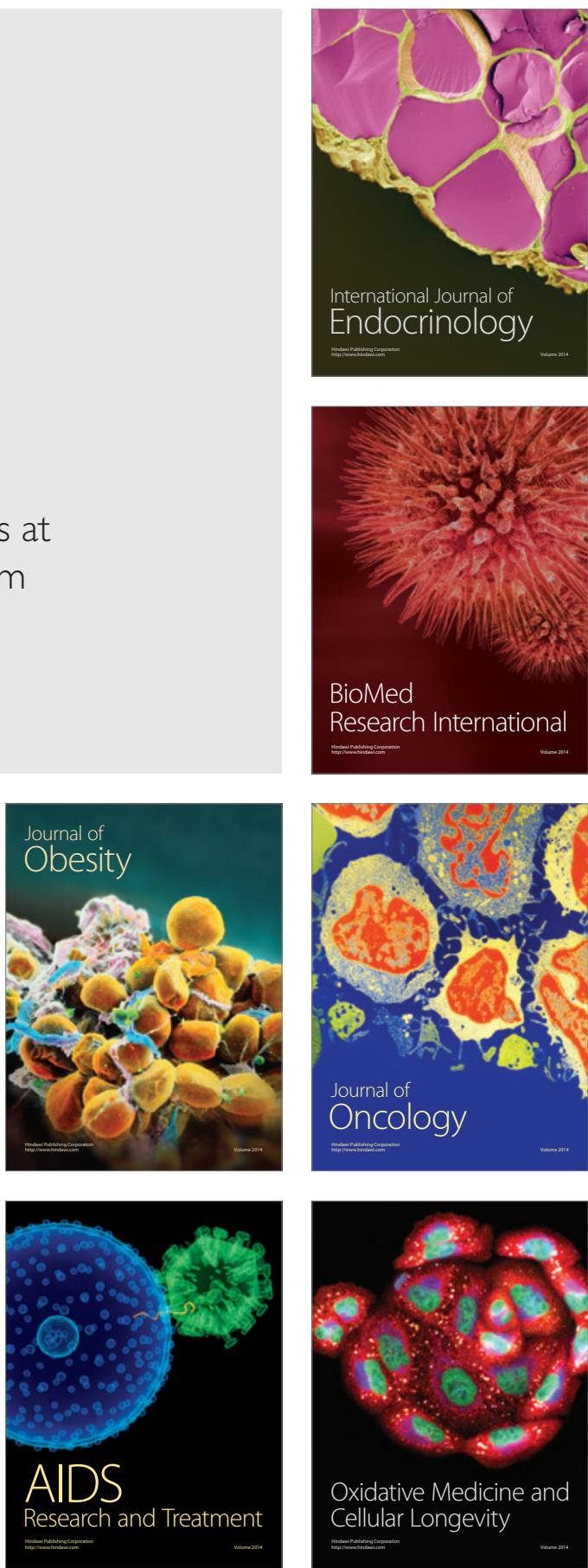